\title{
Incorporación de harina de amaranto para la obtención de bocaditos de carne con bajo contenido de grasa
}

\section{(Incorporating amaranth flour to obtain low-fat meat snacks)}

\author{
Melisa, G. Britez"1, Fabiana A. Rolhaiser ${ }^{1,2}$, Ana M. Romero1, Mara, C. Romero ${ }^{1,2}$
}

\begin{abstract}
Resumen
El objetivo de este trabajo fue evaluar el efecto de la incorporación de harina de amaranto a bocaditos de carne con bajo contenido de grasa, mediante análisis sensorial y valor nutricional. Para ello se elaboró un control y tres formulaciones, en las cuales se varió en la forma de incorporar la harina de amaranto. Las unidades de muestra se moldearon en forma de bocaditos de $50 \pm 0.50 \mathrm{~g}$ y se cocinaron en un horno eléctrico, hasta alcanzar una temperatura interna de $72{ }^{\circ} \mathrm{C}$ en el centro térmico de las muestras, para garantizar la calidad microbiológica. La evaluación sensorial de las muestras se realizó inmediatamente después del proceso de cocción, empleando una escala hedónica de 5 puntos. Los atributos evaluados fueron: olor, color, sabor, terneza, jugosidad, aceptación general e intención de compra. Además, se analizó el rendimiento a la cocción y la composición proximal de los productos elaborados y esto se comparó con la información nutricional de un producto comercial. Las distintas formulaciones elaboradas, tuvieron buena aceptación por parte de los evaluadores, quienes enunciaron, además, la intención de compra de las mismas, lo que resultó de la apreciación general de cada muestra. La forma en la que se incorporaron los ingredientes, afectó al rendimiento y a la composición proximal de las formulaciones ensayadas, lo cual puede atribuirse a la retención de fluidos durante la cocción. Los bocaditos elaborados presentaron, asimismo, menores contenidos de grasa y de sodio en comparación con una muestra comercial, lo que los hace más saludables.
\end{abstract}

\section{Palabras clave}

Harina sin gluten; producto cárnico funcional; valor nutricional; sodio; evaluación sensorial.

\begin{abstract}
The aim of this work was to evaluate the effect of incorporation of amaranth flour into meat snacks with low fat content by sensory analysis and nutritional value. For this, a control and three formulations were developed, varying the way in which the amaranth flour was incorporated. Samples units were molded into $50 \pm 0.50 \mathrm{~g}$ snacks and cooked in an electric oven until reaching an internal temperature of $72{ }^{\circ} \mathrm{C}$ in the thermal center of samples to guarantee microbiological quality. Sensory evaluation of the samples was carried out immediately after cooking process, using a 5-point hedonic scale. The attributes evaluated were odor, color, flavor, tenderness, juiciness, general acceptance and purchase intention. In addition, cooking yield and proximal composition of the elaborated products were analysed being the nutritional information compared with the obtained from a commercial product. The different formulations developed were well accepted by the evaluators, who also stated the purchase intention, which results from the general appreciation of each sample. The method in which ingredients were incorporated affected cooking yield and proximal composition of formulations, which can be attributed to a greater or lesser retention of fluids during cooking, in consequence cooking yield should be increased. The snacks produced also had lower fat and sodium content compared to a commercial sample, which makes them healthier.
\end{abstract}

\section{Keywords}

gluten-free flour; functional meat product; nutritional value; sodium; sensory evaluation.

\section{Introducción}

La carne es tradicionalmente reconocida como un alimento altamente nutritivo, no solo por su contenido de proteínas y aminoácidos esenciales, sino también porque es fuente de vitamina

1 Universidad Nacional del Chaco Austral, Pcia. Roque S. Peña, Chaco, Argentina (melisabritez@uncaus.edu.ar; fabianarolhaiser@uncaus.edu.ar; amr@uncaus, edu.ar; mara@uncaus.edu.ar).

2 INIPTA-CONICET. Pcia. Roque S. Peña, Chaco, Argentina. 
B12 y de minerales importantes, como el zinc y el hierro, este último con una biodisponibilidad que no se altera por la cocción (Olmedilla y Jiménez-Colmero, 2014). Pero, en general, contiene un alto porcentaje de ácidos grasos saturados y colesterol, y existe una relación entre el consumo de este tipo de grasas animales y un mayor riesgo de algunos trastornos, como las enfermedades coronarias (Rodríguez-Carpena, Morcuende y Estévez, 2012).

Además, el aporte de hierro de la carne en las personas es primordial, no solo para producir glóbulos rojos sanos, que transportan el oxígeno a los tejidos, sino también para obtener un buen desarrollo cerebral. Es así que su carencia puede generar desde anemia hasta un desarrollo cognoscitivo incompleto, lo cual puede poner en riesgo la salud de gran número de personas. Debido a esto, el Ministerio de Salud Pública de Argentina, (2016) recomienda consumir carnes rojas hasta tres veces por semana y se representa la porción de la misma por el tamaño de la palma de la mano.

Entre los productos cárnicos más consumidos por los niños, se encuentran los bocaditos, medallones, formitas o nuggets de carne, los que pueden ser adquiridos en las góndolas de los supermercados. Sin embargo, los ingredientes más utilizados en estos productos son el pan rallado o harina de trigo, lo que los hace inaccesibles para un grupo de consumidores como los enfermos celíacos, pues contienen, además, un alto porcentaje de grasas, generalmente saturadas.

En los últimos años, la industria viene desarrollando productos cárnicos con bajo contenido de grasa, generalmente mediante el uso de ingredientes tales como aceites vegetales y extractos naturales, productos vegetales y fibra, para mejorar las propiedades funcionales y la calidad y estabilidad del producto (Shahiri Tabarestani y Mazaheri Tehrani, 2014). Sin embargo, el desarrollo de productos cárnicos con menor contenido de grasa no solo implica una reducción de los rendimientos de cocción (R), por la pérdida de grasa y humedad durante este procedimiento, sino que representa también la pérdida de características cualitativas importantes para la aceptación por parte de los consumidores.

Para evitar dichas pérdidas se emplean aditivos como las proteínas de origen vegetal (soja), otras harinas como la de quinua o arroz, lo cual mejora el ligado del agua durante el procesamiento de las materias primas, aumenta la estabilidad de la mezcla y mejora las propiedades sensoriales del producto disminuyendo la pérdida de características cualitativas (López, Botero y Arias, 2016; Moreno Vaca y Maldonado Pacheco, 2015; Pérez Touzón, Hernández et al., 2018). Así, dependiendo de los ingredientes utilizados en la formulación de estos productos, los mismos pueden ser concebidos como alimentos funcionales, dado que no solo proporcionan nutrientes de reconocidos efectos beneficiosos y nutricionales básicos en el organismo de las personas, sino que ayudan a reducir el riesgo de sufrir determinadas enfermedades (Funtes, Acevedo y Gelvez, 2015).

El amaranto es un pseudocereal de origen andino-americano, que posee un elevado contenido de proteínas (18\%) de alto valor biológico, por la calidad de sus aminoácidos (como por ejemplo la lisina que es esencial en la alimentación humana), un importante contenido de fibra (10 \%), un bajo contenido de grasa (5\%), un alto nivel de hierro (90 ppm), y sustancias antioxidantes como tocoferoles y tocotrienoles (Herrera y Montenegro, 2012). La incorporación de harina de amaranto (HAo), garantizaría la presencia de nutrientes de alto valor nutricional y constituiría una buena alternativa para la sustitución de harina de trigo o pan rallado en los bocaditos de carne comerciales. Además, teniendo en cuenta que estos alimentos son aceptados por los consumidores de todas las edades, se vuelven una alternativa interesante y excelente oportunidad para transformar los productos cárnicos tradicionales, ya que en la actualidad la 
harina de amaranto no es utilizada como ingrediente funcional en productos cárnicos (Administración Nacional de Medicamentos, Alimentos y Tecnología Médica, [Anmat], 2018).

Como las cantidades y formas de incorporación de ingredientes a los sistemas cárnicos, además de afectar la composición de los mismos, pueden incidir también sobre las propiedades tecnológicas y organolépticas de estos sistemas, la industria recurre al análisis sensorial. Estos ensayos se utilizan para determinar el grado de aceptación de un producto por parte del consumidor, y generalmente indican la intención de compra real y de la ingesta del mismo (Pintado, Herrero, Ruiz-Capillas et al., 2015; Pintado, Herrero, Jiménez-Colmenero, Ruiz-Capillas, 2016; Romero, Fogar, Rolhaiser et al., 2018; Singh-Ackbarali y Maharaj, 2014). Así, en este trabajo se plantea evaluar el efecto de la incorporación de harina de amaranto a bocaditos de carne con bajo contenido de grasa, mediante análisis sensorial y valor nutricional.

\section{Metodología}

\subsection{Ingredientes}

Los productos a desarrollar se prepararon con: carne vacuna magra, cloruro de sodio (1\%), aceite de girasol y oliva (Aceitera General Deheza, S. A.), harina de amaranto (Celandrina, Praga S. R. L), tripolifosfato de sodio (Sigma-Aldrich), gelatina sin sabor (Arcor), huevo y condimento para milanesa (Alicante, La Virginia S. A.). El producto cárnico fue elaborado en la Planta Piloto que posee la cátedra de Industrias Alimentarias II del Departamento de Tecnología, en la Universidad Nacional del Chaco Austral, bajo estrictas normas de higiene y buenas prácticas de manufactura.

\subsubsection{FORMULACIONES DE PRODUCTOS CÁRNICOS ENSAYADAS}

Siguiendo el procedimiento indicado por Pintado, Herrero, Jiménez-Colmenero y Ruiz-Capillas (2016), se elaboraron 4 formulaciones, denominadas C (control), F1, F2, F3, cuyas composiciones se muestran en la tabla 1.

Tabla 1. Formulaciones de productos cárnicos (bocaditos/nuggets) ensayadas (g)

\begin{tabular}{|l|c|c|c|c|}
\hline \multicolumn{1}{|c|}{ Ingredientes } & C & F1 & F2 & F3 \\
\hline Carne & 670 & 670 & 670 & 670 \\
\hline Sal & 10 & 10 & 10 & 10 \\
\hline Agua & 100 & 60 & 0 & 0 \\
\hline Aceite & 200 & 200 & 0 & 0 \\
\hline HAo & 0 & 40 & 0 & 0 \\
\hline Emulsión de HAo & 0 & 0 & 305 & 0 \\
\hline Gel de HAo & 0 & 0 & 0 & 305 \\
\hline TPP & 5 & 5 & 0 & 0 \\
\hline Condimento para milanesa & 10 & 10 & 10 & 10 \\
\hline Rebozado de HAo & 5 & 5 & 5 & 5 \\
\hline Total & 1000 & 1000 & 1000 & 1000 \\
\hline
\end{tabular}

La variación en las formulaciones F1, F2 y F3 radicó en la forma en la que la harina de amaranto, como ingrediente, fue incorporada a la matriz cárnica. Así, para la formulación F1 la harina de amaranto fue agregada directamente a la pasta cárnica con el resto de los componentes; para F2 la HAo fue incluida en la carne picada a través de una emulsión con agua, 
aceite, y el emulsionante tripolifosfato de sodio (TPP), la cual fue preparada por agitación con minipimer durante 10 minutos (con descansos de 15 segundos hasta completar el tiempo) y luego incorporada. Y, por último, para F3 la harina de amaranto fue añadida en forma de una emulsión gelificada, formulada con agua, aceite, HAo y TPP (mezcla enriquecida con harina de amaranto empleando proteína de gelatina como agente gelificante). Esta última formulación fue seleccionada de acuerdo con los resultados obtenidos en un estudio previo (Rolhaiser, Coronel, Michaluk et al. 2019). Además, en todas las formulaciones la harina de amaranto fue usada como rebozador.

\subsection{Elaboración del producto cárnico}

La carne fue previamente cortada en fragmentos, eliminándose grasa y tejido conectivo visible, luego se procesó empleando una picadora manual (Philips @ 7627, 650 watts), durante dos minutos, para obtener una pasta homogénea. Todos los ingredientes de cada formulación se pesaron en cantidades tecnológicamente adecuadas y luego, según correspondiese, se mezclaron en recipientes durante cinco minutos para eliminar la mayor cantidad de aire y formar una mezcla integrada. Se elaboró un total de veinte muestras para cada formulación presentada en la tabla 1.

En otro recipiente se batieron los huevos con sal y condimento para milanesa; esta mezcla se utilizó para pincelar la pasta y luego realizar el rebozado del alimento cárnico, con la harina de amaranto (HAo), para la obtención del nugget. Para todos los ensayos las unidades de muestra se moldearon en forma de bocaditos de $50 \pm 0.50 \mathrm{~g}$ y se cocinaron en un horno eléctrico (200 ${ }^{\circ} \mathrm{C}$ ) hasta alcanzar una temperatura interna de $72{ }^{\circ} \mathrm{C}$ en el centro térmico de las muestras, para garantizar la calidad microbiológica. La temperatura interna se controló utilizando un termómetro de punción (Testo modelo 925, Lenzkirch, Alemania).

\subsection{Evaluación sensorial de los productos elaborados}

Teniendo en cuenta que la aceptación general es un factor crucial cuando se evalúa la incorporación de un nuevo ingrediente en un producto alimenticio, el ensayo se realizó con un panel de 100 personas no entrenadas, pertenecientes a personal que trabaja o estudia en la Universidad Nacional del Chaco Austral (profesores y alumnos, entre 18 y 60 años), quienes aceptaron voluntariamente participar. Antes de comenzar el ensayo, los jueces dispuestos en forma separada uno del otro por medio de cabinas portátiles previamente armadas, fueron informados sobre los ingredientes empleados en el producto, para prevenir alguna intolerancia y/o algún tipo de alergia a los componentes utilizados en su elaboración por parte de los participantes (Schrotlin y Secchi, 2018).

Las formulaciones ensayadas fueron evaluadas inmediatamente después del proceso de cocción, disponiendo para ello las muestras en platos blancos de plástico junto con 1 galletita sin sal y 1 vaso de agua. Junto con las formulaciones elaboradas se presentó a los panelistas una muestra comercial (adquirida en un comercio de la zona) y las muestras (codificadas con números de tres cifras) fueron exhibidas en forma aleatoria (Sampere, Sánchez, Valerias et al., 2014). Los atributos evaluados fueron: olor, color, sabor, terneza, jugosidad, aceptación general e intención de compra, empleando una escala hedónica de 5 puntos, desde la categoría 1 "me disgusta mucho" hasta la 5 "me gusta mucho" (Gao, Zhang, Zhou, 2014). Además de la escala, 
tuvieron un espacio en la ficha de respuestas (observaciones) para expresar alguna particularidad que consideraran relevante.

\subsection{Rendimiento y análisis de la composición proximal de los productos}

El rendimiento de los productos luego de la cocción se calculó según la Ecuación (1), lo que informa sobre las propiedades para ligar agua y grasa. Se efectuó además el análisis proximal, para la determinación del valor nutricional de las muestras; se determinó humedad, proteínas, grasas y cenizas, según técnicas oficiales de la Association of Official Analytical Chemists, (AOAC), 1998, empleando los métodos 950.46, 991.36, 960.52, y 920.153, respectivamente para dichos nutrientes. Los carbohidratos se determinaron por el método de antrona de Glegg, (1956); el sodio se determinó por fotometría de llama, método AOAC 985.35 y el valor energético siguiendo la metodología oficial del Código Alimentario Argentino (CAA), (2005)). Para el cálculo de proteínas se empleó el factor de conversión 6.25.

$$
\text { Rendimiento }(R \%)=\left[\frac{\text { peso nugget cocido }(g)}{\text { peso nugget crudo }(g)}\right] \times 100
$$

Ecuación (1)

Todas las determinaciones realizadas se hicieron por duplicado para cada formulación.

Con objeto de comparar el valor nutricional de las muestras se adquirió un producto comercial (bocadito de carne). La comparación se efectuó a través de lo reportado por el fabricante en la etiqueta nutricional del producto. Según la declaración nutricional, el mismo se elaboró con carne vacuna, pan rallado, agua, huevo, aceite vegetal, almidón, proteína aislada de soja, sal, estabilizante, resaltador del sabor y antioxidante.

\subsection{Análisis de datos}

Los datos obtenidos de la evaluación sensorial, rendimiento y composición proximal se recogieron como la media \pm la desviación estándar, de las determinaciones realizadas a cada muestra, y se analizaron con el programa Statgraphics Plus bajo entorno de Windows, mediante el análisis de la varianza (Anova simple) y mediante comparaciones múltiples, empleando el test HSD de Tukey, con un nivel de significancia del $5 \%(p \leq 0,05)$ entre los productos analizados.

\section{Resultados}

\subsection{Evaluación sensorial del producto realizado}

La figura 1 muestra los resultados obtenidos en la evaluación sensorial de los productos realizados. Para las muestras elaboradas con harina de amaranto, F3 obtuvo el mayor puntaje en los atributos sabor (3.86 \pm 0.03 ), jugosidad (3.66 \pm 0.02$)$ y terneza $(4.06 \pm 0.013)$, diferenciándose de las demás formulaciones ensayadas $(p<0.05)$. La mayor valoración de estas propiedades en la formulación 3, puede atribuirse a la consistencia que le proporcionó a la misma la emulsión agua-aceite-HAo gelificada adicionada, que no solo posibilitó la incorporación de agua al producto, sino que permitió su retención durante la cocción. No ocurrió esto último en las demás formulaciones que presentaron mayor dureza y menor jugosidad.

Aunque la formulación F1 en la que HAo se incorporó directamente a la preparación, fue observada por los evaluadores por su consistencia blanda en el interior y su firmeza externa. 
Cuando se analizaron los datos obtenidos, no se encontró diferencias significativas entre esta, la formulación F2, y la muestra control en la evaluación de la terneza ( $p>0.05$ ). Respecto a los otros atributos (color, aceptabilidad general e intención de compra), de las muestras con harina de amaranto como ingrediente y el control, todas tuvieron una buena valoración (con puntajes mayores a 3.30) y no se observaron diferencias significativas entre las mismas.

Figura 1. Evaluación sensorial de las formulaciones elaboradas.

Donde C: contiene harina de amaranto como rebozador; F1: contiene HAo incorporada a la masa; F2: contiene emulsión formada con agua-aceite-HAo; F3 contiene una emulsión agua-aceite- HAo gelificada

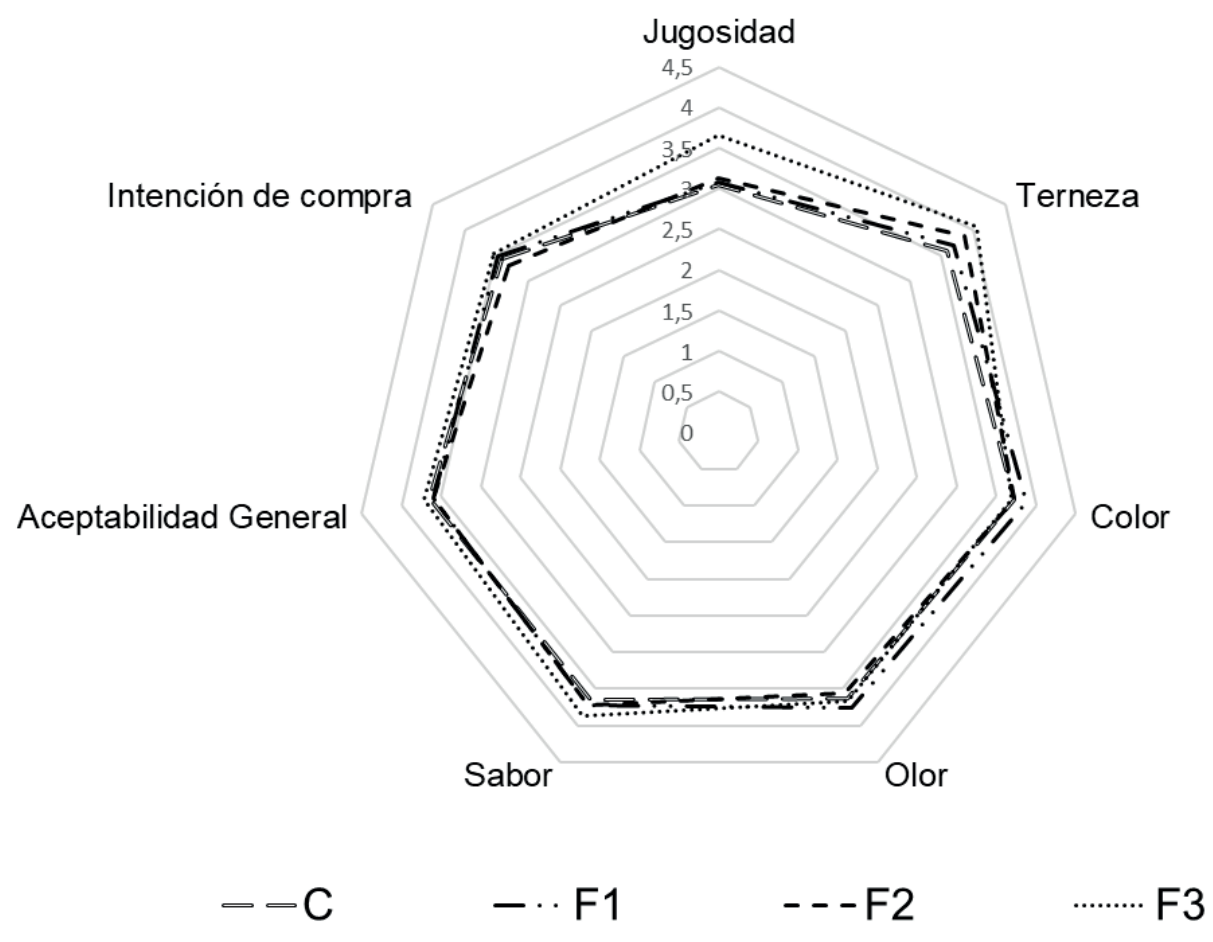

\subsection{Evaluación del rendimiento de cocción y composición proximal del producto cárnico}

Los rendimientos obtenidos y los resultados para la composición nutricional de los productos desarrollados se presentan en la tabla 2. Alli es posible observar que si bien todas las formulaciones que fueron elaboradas con HAo como ingrediente (F1, F2 y F3), presentaron mayores rendimientos en la cocción que la muestra control (que solo contenía HAo como rebozador). La muestra F1 en la que este componente fue incorporado directamente a la pasta cárnica mostró

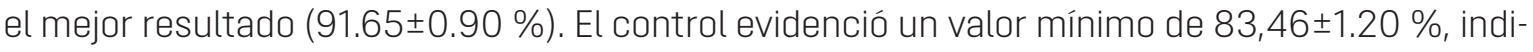
cando una menor retención de fluidos (agua o grasa) durante la cocción. 
Tabla 2. Composición proximal de las formulaciones ensayadas

\begin{tabular}{|l|l|l|l|l|}
\hline \multicolumn{1}{|c|}{ Determinaciones } & \multicolumn{1}{c|}{ Control } & \multicolumn{1}{c|}{ F1 } & \multicolumn{1}{c|}{ F2 } & \multicolumn{1}{c|}{ F3 } \\
\hline Rendimientos $(\%)$ & $83.46 \pm 1.20^{\mathrm{a}}$ & $91.65 \pm 0.90^{\mathrm{d}}$ & $89.17 \pm 0.96^{\mathrm{c}}$ & $86.06 \pm 0.87^{\mathrm{b}}$ \\
\hline Humedad $(\mathrm{g} / 100 \mathrm{~g})$ & $48.36 \pm 1.62^{\mathrm{a}}$ & $54.93 \pm 1.35^{\mathrm{b}}$ & $53.76 \pm 0.39^{\mathrm{b}}$ & $60.75 \pm 0.94^{\mathrm{c}}$ \\
\hline Grasa total $(\mathrm{g} / 100 \mathrm{~g})$ & $12.15 \pm 0.61^{\mathrm{b}}$ & $12.33 \pm 0.85^{\mathrm{b}}$ & $11.59 \pm 0.23^{\mathrm{b}}$ & $6.68 \pm 0.19^{\mathrm{a}}$ \\
\hline Proteínas $(\mathrm{g} / 100 \mathrm{~g})$ & $20.26 \pm 0.05^{\mathrm{ab}}$ & $22.31 \pm 0.94^{\mathrm{b}}$ & $19.40 \pm 0.05^{\mathrm{a}}$ & $19.81 \pm 0.10^{\mathrm{ab}}$ \\
\hline Carbohidratos $(\mathrm{g} / 100 \mathrm{~g})$ & $17.19 \pm 0.08^{\mathrm{a}}$ & $19.12 \pm 0.67^{\mathrm{b}}$ & $22.88 \pm 0.37^{\mathrm{c}}$ & $17.08 \pm 0.20^{\mathrm{a}}$ \\
\hline Cenizas $(\mathrm{g} / 100 \mathrm{~g})$ & $3.32 \pm 0.18^{\mathrm{b}}$ & $3.08 \pm 0.03^{\mathrm{b}}$ & $3.42 \pm 0.16^{\mathrm{b}}$ & $2.49 \pm 016^{\mathrm{a}}$ \\
\hline Sodio $(\mathrm{mg} / 100 \mathrm{~g})$ & $224.84 \pm 9.06^{\mathrm{a}}$ & $327.25 \pm 4.00^{\mathrm{b}}$ & $358.58 \pm 2.99^{\mathrm{c}}$ & $225.96 \pm 3.01^{\mathrm{a}}$ \\
\hline
\end{tabular}

*Las referencias de las muestras se encuentran en la tabla 1. Los resultados están expresados como la media \pm desviación estándar. Diferentes letras en la misma fila indican diferencias significativas.

Cuando se analizaron los contenidos de humedad y de grasa, todas las muestras con harina de amaranto incorporada a la matriz exhibieron valores mayores de humedad que el control; mientras que no hubo diferencias significativas entre el control, F1 y F2 en el porcentaje de grasa después del calentamiento, observándose solo un comportamiento claramente distinto en relación a este parámetro para la muestra F3, en la que la HAo fue incluida en la matriz en forma de emulsión gelificada. Así, F3 fue la que presentó el contenido de humedad más alto $(p<0.05)$ y el contenido de grasa más bajo $(p>0,05)$, entre las formulaciones analizadas.

En cuanto a los resultados obtenidos para proteínas y carbohidratos, F1 presentó el mayor contenido de proteínas y un alto valor en carbohidratos, probablemente debido a la pérdida de agua del producto durante su cocción, mientras que F2 mostró un mayor porcentaje de hidratos de carbono $(p<0.05)$. La incorporación de harina de amaranto emulsionada podría haber favorecido la disponibilidad de los carbohidratos en el producto. El menor contenido de cenizas fue observado para F3 $(p<0.05)$.

Los niveles de sodio de todas las formulaciones ensayadas estuvieron comprendidos entre 256 y 391 mg/100 g, todos ellos cumplen con la Ley 26.905 (2013), promovida por el Ministerio de Salud de Argentina y cuyo objeto es lograr la reducción del consumo de sodio en la población en Argentina.

La muestra comercial, bocadito de carne, de acuerdo a la información nutricional del rótulo del producto presentó una mayor proporción de los macronutrientes proteínas, grasas e hidratos de carbono ( $25 \mathrm{~g} / 100 \mathrm{~g}, 14 \mathrm{~g} / 100 \mathrm{~g}$ y $33 \mathrm{~g} / 100 \mathrm{~g}$ respectivamente). Cabe destacar que su nivel de sodio (800 mg/100 g) fue marcadamente superior al de las formulaciones desarrolladas con harina de amaranto.

\section{Discusión}

Durante la evaluación sensorial de los productos elaborados con harina de amaranto, los evaluadores informaron una aceptabilidad positiva, debido a la textura y jugosidad de los mismos, especialmente de la muestra F3, en la que la forma de incorporación de este ingrediente fue más efectiva en ligar y retener el agua después de la cocción, teniendo en cuenta también el contenido de fibra de la harina. Esto concuerda con lo que informaron Massari, Plencovich y Trouilh Zarza, (2017), en su trabajo referido a la elaboración de scones con harina de amaranto, 
donde se reportó la buena aceptabilidad de los productos en lo que respecta especialmente al sabor y la textura de los mismos.

Pérez Touzón, Hernández et al., (2018), encontraron que la evaluación sensorial calificó el producto entre bueno y muy bueno a un producto cárnico elaborado con harina de arroz; harina de trigo y fécula de papa, y mostraron que las cantidades de ingredientes adicionados ejercen un importante efecto en las características cualitativas del producto. Del mismo modo, investigadores demostraron que la inclusión de harina de garbanzo y gel de Aloe vera, como ingredientes, modifican levemente las características sensoriales del producto, mejorándolas en comparación con las de un control (Acevedo Correa, Montero Castillo, Atencio Salas et al. 2017). Respecto del color, no se observaron diferencias significativas entre las distintas formulaciones ensayadas ( $p>0.05)$. Además, en general, las muestras con harina de amaranto presentaron un color más oscuro después de la cocción. Esto concuerda con lo reportado por Guzmán Urrutia y López Lemus (2015), para galletas elaboradas con harina de amaranto, en las que, al aumentar la proporción de la harina, las galletas tendían a ser más oscuras.

El rendimiento de cocción indica la cantidad de agua absorbida durante este proceso. Las formulaciones ensayadas presentaron un mayor rendimiento que el control $(p<0.003)$, lo cual concuerda con lo hallado por Argel, Ranalli, Califano y Andrés (2017), en productos procesados de cerdo de bajo contenido lipídico, adicionados con aceite vegetal y harinas de legumbres. F3 presentó el mayor contenido de humedad, pero F1 presenta el mayor rendimiento debido a la absorción de agua por parte de la harina de amaranto y, además, retuvo mejor la grasa, lo que también incidió en una menor pérdida de peso y por lo tanto en un mejor rendimiento de cocción. Esto coincide con lo informado por Aslinah, Yusoff y Ismail-Fitry (2018), y Torres, Montero y Martelo (2018) en productos cárnicos elaborados con harinas de frijoles y garbanzos respectivamente.

La forma en la que se incorporaron los ingredientes afectó el contenido de grasa y de hidratos de carbono en la composición proximal de las formulaciones ensayadas, lo que puede atribuirse a una mayor o menor retención de fluidos en los productos y a la contribución de nutrientes realizada por la harina (Romero, Fogar, Rolhaiser et al., 2018), ya que el contenido de carne se mantuvo constante en todas las formulaciones. El contenido de grasa fue menor para F3 respecto de C, F1 y F2, debido a las pérdidas durante la cocción, aunque F3 presentó el mayor contenido de humedad con respecto al resto de las formulaciones, probablemente debido a la incorporación de la emulsión gelificada, lo que fue corroborado en el análisis sensorial como la muestra de mayor jugosidad y terneza.

Asimismo, la muestra F3 evidenció un contenido de carbohidratos totales disponibles, comparable al del control, que solo tenía harina de amaranto como rebozador y un menor porcentaje al de las otras formulaciones, en donde este componente se usó como ingrediente. Respecto a los niveles de proteínas, no se presentaron diferencias significativas entre las formulaciones y el control sin HAo como ingrediente, lo que indicaría que la carne fue responsable de la totalidad de su aporte en las mismas. Estos resultados en general concuerdan a lo informado por Nagai (2018), quien indicó que el agregado de la harina de Undaria sp. permitió el desarrollo de productos cárnicos tipo hamburguesas, con aceite preemulsificado y con menor contenido de sodio respecto a los de consumo cotidiano, con buenas características fisicoquímicas. Similares resultados fueron informados por Santhi y Kalaikannan (2014), en productos elaborados con harina de avena.

El bocadito de carne comercial de acuerdo al rótulo del envase presentó un valor marcadamente superior de carbohidratos que las muestras ensayadas y además, como contiene 
albúmina de huevo en polvo entre sus componentes (aditivo generalmente empleado en la industria), de acuerdo a lo informado en el rótulo, mostró un contenido de proteínas algo superior. Como puede observarse, todas las formulaciones analizadas presentaron menos contenidos de grasa y de sodio que el bocadito comercial, por lo que podrían ser consideradas como alimentos saludables y bajos en sodio, según el Código Alimentario Argentino (2005).

\section{Conclusiones y recomendaciones}

Los resultados obtenidos en estos ensayos indicaron que la harina de amaranto, incorporada al producto de diferentes maneras, podría emplearse en la formulación de bocaditos de carne de bajo tenor graso y reducido contenido de sodio, ya que presentaron buena aceptación sensorial, exhibiendo F3 una mayor jugosidad, terneza, sabor y aceptabilidad general que el control.

Se debe considerar que la estrategia de incorporación de HAo afectó el rendimiento en la cocción y el valor nutricional de los productos elaborados, por lo que estos deben ser mejorados. La formulación con harina de amaranto incorporada como ingrediente directamente a la pasta cárnica (F1) presentó el mayor rendimiento en la cocción, un mayor contenido graso y de carbohidratos que las demás formulaciones; mientras que la muestra F3, en donde la harina de amaranto fue añadida en una emulsión gelificada, brindó un producto de moderado rendimiento pero de bajo contenido de grasa y de sodio, y a su vez con valores adicionales de aceptación en lo que refiere a jugosidad y terneza. El producto comercial presentó mayores contenidos de todos los macronutrientes y también de sodio.

Aunque se deberían llevar a cabo estudios adicionales sobre perfil de ácidos grasos y sobre la calidad microbiológica y oxidativa del producto durante el almacenamiento, los resultados de esta investigación podrían ser útiles para la elaboración de otros productos cárnicos con harina de amaranto o su desarrollo a escala industrial en productos alimenticios de consumo masivo, y que puedan ser accesibles a una población con requerimientos nutricionales específicos, como lo son los enfermos celiacos.

\section{Agradecimientos}

Agradecemos a la Universidad Nacional del Chaco Austral por permitirnos llevar a cabo la investigación.

\section{Bibliografía}

Acevedo Correa, D.; Montero Castillo, P.; Atencio Salas, M. et al. (2017). Elaboración de un producto cárnico tipo salchicha con incorporación de harina de garbanzo y gel de Aloe vera. alimentech Ciencia y Tecnología Alimentaria, 15(1), 5-16.

Administración Nacional de Medicamentos, Alimentos y Tecnología Médica (Anmat). (2018). Nuevo listado de productos aptos para celíacos. Recuperado de http://www.anmat.gov.ar/Alimentos/ libres_gluten/Alimentos_Libres_de_Gluten.asp

Argel, N.; Ranalli, N.; Califano, A. N. y Andrés, S. C. (2017). Incorporación de harinas de legumbres en medallones de carne porcina. La Industria Cárnica Latinoamericana. 206, 36-40.

Aslinah, L. N. F.; Yusoff, M. M. y Ismail-Fitry, M. R. (2018). Simultaneous Use of Adzuki Beans (Vigna angularis) Flour as Meat Extender and Fat Replacer in Reduced-Fat Beef Meatballs (Bebola daging). Journal of Food Science and Technology. doi: 10.1007/s13197-018-3256-1 
Association of Official Analytical Chemists (AOAC). (1998). Official Methods of Analysis (16. . ed.). Washington DC, USA: Horowitz.

Clegg, K. M. (1956). The application of the Anthrone Reagent to the Estimation of Starch in Cereals. Journal of Science of Food and Agriculture, 7:40-44.

Código Alimentario Argentino. (2005). Capítulo V: Normas para la rotulación y publicidad de los alimentos. Resolución Conjunta SPRyRS 149/2005 y SAGPyA 683/2005. Disponible en: http://www. anmat.gov.ar/alimentos/codigoa/capitulo_v.pdf http://www.conal.gob.ar/ultimas_modificaciones/Capitulo_V.pdf

Pérez Touzón, J.; Hernández, U.; Brito, Y. y Lezcano, A. (2018). Efecto de la harina de arroz sobre la calidad de un embutido tipo mortadela. Ciencia y Tecnología de Alimentos, 28(3), 1-6.

Funtes, L.; Acevedo, D. y Gelvez, V. (2015). Alimentos funcionales: Impacto y retos para el desarrollo y bienestar de la sociedad colombiana. Biotecnología en el sector agropecuario y agroindustrial, 13(2), 140-149.

Gao, X.; Zhang, W. y Zhou, G. (2014). Effects of Glutinous Rice Flour on the Physiochemical and Sensory Qualities of Ground Pork Patties. LWT - Food Science and Technology; 58, 135-141. doi: 10.1016/j. Iwt.2014.02.044

Guzmán Urrutia, M. P. y López Lemus, P. G. (2015). Propuesta de formulación de galletas elaboradas con harina compuesta de Amaranthus cruentus (Amaranto) y Sorghum bicolor. Bachelor Thesis. Universidad de El Salvador

Herrera, D. S. y Montenegro, A. (2012). El Amaranto: prodigioso alimento para la longevidad y la vida. Kalpana; 8, 50-66.

López, M. L. M.; Botero, M. J. y Arias, D. F. (2016). Obtención y evaluación fisicoquímica de la harina de cáscara de gulupa (Passiflora edulis sims fo edulis) para su uso en carnes de hamburguesa. La industria cárnica latinoamericana; 195, 56-61.

Massari, T. N.; Plencovich, R. P. y Trouilh Zarza, D. B. (2017). Harina de amaranto y semillas de lino en la elaboración de Scones: aportando aminoácidos y ácidos grasos esenciales. Trabajo de investigación para la Licenciatura en Nutrición. Universidad Nacional de Córdoba, Córdoba.

Ministerio de Salud de la Nación. Guías Alimentarias para la Población Argentina, Buenos Aires, Argentina. (2016). Recuperado de: http://www.msal.gob.ar/images/stories/bes/graficos/0000001007 cnt-2017-06_guia-alimentaria-poblacion-argentina.pdf

Presidencia de la Nación. Boletín Oficial Ley Nacional de Promoción de la Reducción del Consumo de Sodio en la Población. Ley 26.905. Recuperado de: http://www.saij.gob.ar/16-nacional-reglamentacion-ley-26905-sobre-promocion-reduccion-consumo-sodio-poblacion-dn201700000162017-01-04/123456789-0abc-610-0000-7102 soterced?utm_source=newslettersemanal\&utm_medium=email\&utm_term=semanal\&utm_campaign=decreto-nacional\# https://www.boletinoficial.gob.ar/detalleAviso/primera/99389/20131216?busqueda=1

Moreno Vaca, A. y Maldonado Pacheco, P. (2015). Efecto de la sustitución de grasa dorsal de cerdo por aceite de aguacate en la calidad de salchichas de pollo tipo suiza. Enfoque UTE, 6(1), 55 - 70.

Nagai, N. F. (2018). Estudio de harinas de algas marinas comestibles y su incorporación en productos cárnicos. Facultad de Ciencias Exactas. Licenciatura en Ciencia y Tecnología de Alimentos. Universidad Nacional de la Plata.

Olmedilla Alonso, B. y Jiménez-Colmero, F. (2014). Alimentos cárnicos funcionales: desarrollo y evaluación de sus propiedades saludables. Nutrición Hospitalaria, 29(6), 1197-1209. doi: 10.3305/ nh.2014.29.6.7389

Pintado, T.; Herrero, A. M.; Ruiz-Capillas, C. et al., (2015). Effects of Emulsion Gels Containing Bioactive Compounds on Sensorial, Technological, and Structural Properties of Frankfurters. Food Science and Technology International, 0(0) 1-14. doi: 10.1177/1082013215577033

Pintado, T.; Herrero, A. M.; Jiménez-Colmenero, F.; Ruiz-Capillas, C. (2016). Strategies for Incorporation of Chia (Salvia hispanica L.) inFfrankfurters as a Health-promoting Ingredient. Meat Science, 114, 75-84. doi: 10.1016/j.meatsci.2015.12.009 
Rodríguez-Carpena, J. G.; Morcuende, D. y Estévez M. (2012). Avocado, Sunflower and Olive Oils as Replacers of Pork Back-Fat in Burger Patties: Effect on Lipid Composition, Oxidative Stability and Quality Traits. Meat Science, 90(1), 106-115. doi: 10.1016/j.meatsci.2011.06.007

Rolhaiser, F.; Coronel, D; Michaluk, A. G. et al. (2019). Hidrogeles enriquecidos con harina de amaranto como posibles sustitutos de grasa en productos cárnicos. Congreso Nacional de Estudiantes de Ingeniería Química. Mendoza, Argentina.

Romero, M. C.; Fogar, R. A.; Rolhaiser, F. et al. (2018). Development of Gluten-free Fish (Pseudoplatystoma corruscans) Patties by Response Surface Methodology. Journal of Food Science and Technology, doi: 10.1007/s13197-018-3106-1

Sampere, S.; Sánchez, N.; Valerias, L. et al. (2014). Desarrollo y aceptabilidad de bocaditos de sabor para adultos mayores. Actualización en nutrición, 15(4), 99-106.

Santhi, D. y Kalaikannan A. (2014). The Effect of the Addition of Oat Flour in Low-Fat Chicken Nuggets. Journal of Nutrition and Food Science, 4(1), 1-4. doi: 10.4172/2155-9600.1000260

Shahiri Tabarestani, H. y Mazaheri Tehrani M. (2014). Optimization of Physicochemical Properties of Lowfat Hamburger Formulation Using Blend of Soy Flour, Split-pea Flour and Wheat Starch as Part of Fat Replacer System. Journal of Food Processing and Preservation, 38, 278-288. doi: 10.1111/j.1745-4549.2012.00774.x

Schrotlin, R. M. y Secchi, C. M. (2018). Producto alimenticio elaborado a base de harina de algarroba y mijo adecuado para personas con intolerancia al gluten. Actualización en nutrición, 19(4), 113-121.

Singh-Ackbarali, D. y Maharaj, R. (2014). Sensory Evaluation as a Tool in Determining Acceptability of Innovative Products Developed by Undergraduate Students in Food Science and Technology at The University of Trinidad and Tobago. Journal of Curriculum and Teaching; 3(1), 10-27.

Torres, L. L.; Montero, P. M., y Martelo, R. J. (2018). Effect of Chickpea Flour Addition (Cicer Arietinum L.) in Cooking Losses During Blanching of Chorizos. Contemporary Engineering Sciences, 11(36), 1781-1787. doi: 10.12988/ces.2018.84170 